\section{Hymenolepis nana: factores asociados a este parasitismo en un área de salud del Sur de España}

\author{
M. Isabel Cabeza, M. Teresa Cabezas, Fernando Cobo, \\ Joaquín Salas y José Vázquez
}

\section{Hymenolepis nana infection: associated factors with this parasitism in a health area of Southern Spain}

Hymenolepis nana is the most common tapeworm in humans; prevalence rates of $0.1 \%-58 \%$ have been reported. The aim of this study was to determine the prevalence in a health area of Southern Spain and identify the demographic variables potentially associated with increased rates of hymenolepiasis in this area. A retrospective study was performed with patients, who had $H$. nana eggs in fecal samples during january 2000 to december 2013. Parasitological diagnosis relied on microscopic detection in concentrated stool samples. During the study period, 73.660 stool samples were analyzed. H. nana eggs were observed in 158 patients (31 female) with a mean age of 18,9 years. The prevalence during the study period was $0,21 \%$ and $61 \%$ of the infected patients had more than one intestinal parasite. In conclusion, the prevalence of parasitism by $H$. nana in our population was higher than the national average and higher in adults than in children due to the characteristics of our population.

Key words: Hymenolepis nana, inmigration, human intestinal infection, saharauis children.

Palabras clave: Hymenolepis nana, inmigración, parasitosis intestinal, niños saharauis.

\section{Introducción}

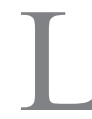

as parasitosis gastrointestinales constituyen un problema de salud mundial, afectando especialmente a países con climas templados y tropicales, donde las condiciones higiénicas no son adecuadas. Hymenolepis nana es un cestodo cosmopolita, siendo el más común en humanos. Se estiman 50-75 millones de portadores en todo el mundo, con una prevalencia global que oscila entre $0,1-58 \%{ }^{1}$.

El ejemplar adulto de $H$. nana (tenia enana) mide entre $15-40 \mathrm{~mm}$ por 0,5-1 mm y consta de escólex, cuello y estróbilo. El número de proglótides oscila entre 150 y 200. Las últimas proglótides, generalmente, se desprenden y se desintegran en el lumen del intestino, eliminándose los huevos con las heces y pudiendo causar autoinfección interna. Los huevos contienen una oncosfera con seis ganchos y está rodeado por una membrana con dos

Hospital de Poniente. El Ejido, Almería, España.

Unidad de Microbiología. Área de Biotecnología (MIC, MTC, FC).

Unidad de Medicina Tropical (JS, JV)

Declaración de intereses: No existe ningún potencial conflicto de interés relacionado con el artículo.

Recibido: 4 de marzo de 2015 / Aceptado: 8 de septiembre de 2015

Correspondencia a:

M. Isabel Cabeza B.

mariaisabel.cabeza@ephpo.es engrosamientos polares, de los cuales surgen de 4 a 8 filamentos. La tenia adulta madura entre 2 a 3 semanas ${ }^{2}$.

Hymenolepis nana, como todos los cestodos, requiere dos tipos de hospederos: el intermediario (dipteros), en el que se desarrolla la larva cisticercoide tras la ingestión de los huevos, y el definitivo, en cuyo intestino delgado se desarrolla el cestodo adulto tras la ingestión de la larva cisticercoide. En esta cestodosis, los hospedadores definitivos (humanos y ratones) pueden actuar también como intermediarios, es decir, son capaces de evolucionar mediante ciclos vitales monoxénicos y heteroxénicos, siendo el primero de ellos el que comúnmente produce la parasitación en el ser humano.

La infección puede tener un impacto epidemiológico importante en las unidades familiares, ya que se puede transmitir directamente entre seres humanos y por autoinfección interna por ingestión de huevos de H. nana, principalmente de heces humanas o por contaminación de los alimentos ${ }^{3,4}$.

Las manifestaciones clínicas dependen del número de parásitos, edad y estado general del individuo afectado. Varían desde infecciones asintomáticas hasta cuadros graves con parasitosis masivas, siendo éstas más frecuentes en niños. Puede llegar a producir anorexia, dolor abdominal, prurito alrededor del ano, irritabilidad y diarrea ${ }^{5}$.

El diagnóstico etiológico se efectúa mediante la observación microscópica de los huevos o proglótides en deposiciones y la correspondiente identificación morfométrica característica de los huevos ${ }^{1}$. El tratamiento de elección es praziquantel en dosis única de $25 \mathrm{mg} / \mathrm{kg}$ por vía oral ${ }^{6,7}$. Niclosamida y nitazoxanida son tratamientos alternativos ${ }^{8,9}$.

Se ha observado un aumento del diagnóstico de estas parasitosis en Europa y en el área de salud del Hospital de Poniente en los últimos años, debido a varios factores, entre los que se encuentran: el gran número de personas inmigrantes procedentes de países en vías de desarrollo con alta prevalencia de parásitos intestinales (Marruecos, Sudáfrica y América del Sur), la acogida de niños saharauis por familias españolas durante los meses de verano, los desplazamientos a estas áreas endémicas y, por otro lado, por el aumento del consumo de alimentos frescos procedentes de estas áreas ${ }^{10-13}$.

El objetivo de este estudio es conocer la prevalencia de hymenolepiasis en nuestra área, así como identificar las variables demográficas potencialmente asociadas a esta parasitosis, ya que los datos obtenidos contribuirán a la comprensión general de la epidemiología de H. nana en nuestra población.

\section{Material y Método}

Se realizó un estudio observacional descriptivo retrospectivo de todos los pacientes del Hospital de Poniente de El Ejido (Almería) cuyas heces fueron remitidas al Servicio de Microbiología y en los que se observaron huevos de H. nana, entre enero de 2000 a diciembre de 2013.

El diagnóstico se realizó mediante la observación al microscopio de una gota de heces concentrada mediante el método de Ritchie modificado. A 29 de estos pacientes se les remitió a la consulta de medicina tropical de nuestro hospital, realizándoles el protocolo de atención al inmigrante con patologías importadas. Este protocolo incluye un análisis de sangre general, con perfil renal y hepático, y un estudio de parásitos en sangre, deposiciones y orina.

Las muestras de sangre se concentraron mediante el test de $\mathrm{Knott}^{14} \mathrm{y}$ las de orina se centrifugaron durante $10 \mathrm{~min}$ a $1.500 \mathrm{rpm}$ y posteriormente se observaron al microscopio. Los parásitos fueron identificados por su morfología. 


\begin{tabular}{|c|c|c|c|c|c|c|c|c|c|c|c|c|c|}
\hline Año & 2001 & 2002 & 2003 & 2004 & 2005 & 2006 & 2007 & 2008 & 2009 & 2010 & 2011 & 2012 & 2013 \\
\hline Prevalencia & 0,31 & 0,03 & 0,03 & 0,16 & 0,12 & 0,27 & 0,47 & 0,24 & 0,21 & 0,27 & 0,24 & 0,20 & 0,13 \\
\hline Casos & 3 & 1 & 1 & 6 & 6 & 11 & 24 & 21 & 20 & 22 & 19 & 14 & 10 \\
\hline Porcentaje & 1,9 & 0,6 & 0,6 & 3,8 & 3,8 & 7,0 & 15,2 & 13,3 & 12,7 & 13,9 & 12,0 & 8,9 & 6,3 \\
\hline Total pacientes & 959 & 3.101 & 2.981 & 3.823 & 5.015 & 4.062 & 5.061 & 8.758 & 9.500 & 8.274 & 7.767 & 6.905 & 7.454 \\
\hline
\end{tabular}

Tabla 2. Distribución mensual de casos de Hymenolepis nana en muestras de deposiciones durante el año. Hospital de Poniente de El Ejido (Almería)

\begin{tabular}{|lccccccccccccc} 
Año & Enero & Febrero & Marzo & Abril & Mayo & Junio & Julio & Agosto & Septiembre & Octubre & Noviembre & Diciembre \\
n de pacientes & 7 & 9 & 7 & 12 & 11 & 21 & 51 & 10 & 6 & 11 & 7 \\
Porcentaje & 4,4 & 5,7 & 4,4 & 7,6 & 7,0 & 13,3 & 32,3 & 6,3 & 3,8 & 7,0 & 4,4 & 3,8 \\
\hline
\end{tabular}

\section{Resultados}

Durante el período de estudio se analizaron las muestras de 73.660 pacientes, siendo $71,2 \%$ de sexo masculino.

Del total de pacientes estudiados, se observaron huevos de H. nana en 158 muestras de deposiciones, de 149 pacientes (siete pacientes tuvieron muestras positivas repetidas). Se obtuvo una prevalencia media de $0,21 \%$ y una prevalencia por años que se muestra en la Tabla 1. El 79,2\% (n: 118) de los pacientes eran de sexo masculino y $20,8 \%$ (n: 31 ) mujeres, con una media de 18,9 (rango 2-51) años de edad. De ellos, 65 (43,6\%) eran niños ( $\leq 15$ años) (Tabla 2$)$.

De los pacientes estudiados, $86,2 \%$ (n: 127) eran procedentes de África; 9,4\% (n: 14) de Sudamérica; 1,3\% (n: 2) de Europa del Este; 1,3\% (n: 2) de Asia; sólo $0,7 \%$ (n: 1) era autóctono y en tres casos (2\%) no constaba su país de origen.

De los 127 pacientes procedentes de África, 53 (35,6\%) eran de Marrue$\cos , 37(24,8 \%)$ pacientes eran originarios del Sahara Occidental dentro del Programa de acogida "Vacaciones en paz" y 37 (24,8\%) de los pacientes eran de otras zonas de África.

El $61,1 \%$ (91) pacientes estaban poliparasitados, de los cuales $23,4 \%$ (n: 37) tenían más de dos parásitos.

Los parásitos intestinales aislados en nuestros pacientes fueron: Entamoeba histolytica/dispar (34\%), Giardia lamblia (25,8\%), Uncinaria (11,3\%), Trichuris trichiura (2,1\%), Schistosoma mansoni $(2,1 \%)$, Enterobius vermicularis $(1 \%)$ y Strongyloides stercoralis (1\%) y con especies no patógenas: Entamoeba coli (41,2\%), Blastocystis spp (33\%).

Estudios realizados en sangre y orina revelaron la presencia de Mansonella perstans (1\%), y Schistosoma haematobium (2,1\%).

Sólo 17,8\% (n: 24) presentaban eosinofilia en el hemograma. En 6,4\% (n: 9) de los pacientes, este dato fue desconocido. Se desconocieron otros datos clínicos.

\section{Discusión}

En la última década, Almería es un área receptora de personas procedentes de países en vías de desarrollo donde el agua está altamente contaminada con materia fecal, lo que es un indicador de salud y de las condiciones ecológicas de estos países ${ }^{15}$. Desde el punto de vista clínico, los estudios epidemiológicos como éste son importantes porque revelan los riesgos asociados con las parasitosis: factores ambientales, socio-económicos y de saneamiento, comportamientos demográficos y los relacionados con la salud que se saben que influyen en la transmisión y distribución de los parásitos intestinales en las comunidades humanas ${ }^{16}$.

Las diferencias de resultados entre los diferentes estudios se atribuyen a variaciones en las condiciones climáticas, la disparidad socio-económica entre países desarrollados y en vías de desarrollo, y la posible falta de resultados epidemiológicos adecuados en ciertas localidades sin protección, lo que se traduce como una invisibilidad de ciertas enfermedades parasitarias y otros problemas de salud ${ }^{17,18}$.

En España, la prevalencia general es de alrededor de $0,1 \%$, y en Almería es el doble con una media de $0,21 \%$, llegando a cuadriplicarse $(0,47 \%)$ en el año 2007, cuando se produjo la mayor afluencia de inmigración. Esta prevalencia es también más alta en verano, influenciada principalmente por el Programa anual "Vacaciones en paz", donde 25\% de los pacientes diagnosticados en este estudio provienen del Sahara Occidental ${ }^{19}$.

Siete pacientes de nuestro estudio tuvieron dos o tres veces la misma parasitosis en diferentes años, debido a que regresaban a sus países de origen (niños saharauis o visitas a familiares), produciéndose así una nueva reinfección al vivir en las mismas condiciones higiénicas. Esto nos debe poner en guardia para repetir el estudio parasitológico cuando estas personas vuelvan de nuevo a nuestro país, para lograr cortar rápidamente la cadena de transmisión.

Más de $60 \%$ de nuestros pacientes presentaban poliparasitismo, afectando a su estado nutricional y de salud. Este estudio pone de relieve las deficiencias en el saneamiento y en la educación para la salud como factores de riesgo en la adquisición de hymenolepiasis y la convivencia frecuente con otros patógenos y parásitos comensales. El principal mecanismo de transmisión de los parásitos intestinales es la vía fecal-oral, incluyendo entre ellos E. histolytica/dispar, Giardia intestinalis, Entamoeba coli y Ascaris lumbricoides. La alta prevalencia de estos parásitos es un importante bioindicador de la persistencia de hábitos higiénicos inadecuados en los campamentos y la contaminación del agua por heces humanas, que también puede aumentar el riesgo de contraer otras enfermedades infecciosas ${ }^{20}$. 
A diferencia de otros estudios, en el nuestro hubo más casos en adultos (53\%) que en niños y más en hombres $(79,2 \%)$ que en mujeres, debido principalmente a las características de la población inmigrante de nuestra zona: varón joven de países en vías de desarrollo que vienen a trabajar a España para enviar dinero para su familia ${ }^{12,15}$.

A la vista de estos resultados, se recomienda el establecimiento de protocolos de atención al inmigrante de estas zonas endémicas, así como a todos los niños procedentes de las mismas en programas de acogida, para una pronta valoración desde su llegada a España. Esto nos permitiría realizar medidas correctivas lo más rápido posible y mejorar así la salud de este grupo, con el fin de evitar una posible transmisión a la población residente. La instauración de estos protocolos sería coste/beneficio favorable en esta población, debido a la alta prevalencia de estos parásitos con respecto al coste de la técnica.

El personal sanitario debe formarse, a través de conferencias educativas y didácticas, sobre la epidemiología de la enfermedad y su modo de transmisión, para que esta población pueda interiorizar que la mejora de sus condiciones de vida y prácticas de higiene evita infecciones y re-infecciones de estos parásitos intestinales, aquí y en su país de origen.

\section{Resumen}

Hymenolepis nana es el cestode más común en humanos, estimándose una prevalencia general que oscila entre $0,1 \%-58 \%$. El objetivo de este estudio fue conocer la prevalencia de esta parasitosis en un área del Sur de España, así como identificar las variables demográficas asociadas a las altas tasas de hymenolepiasis en esta área. Se realizó un estudio retrospectivo de pacientes con presencia de huevos de H. nana en deposiciones, entre enero de 2000 a diciembre de 2013. El diagnóstico parasitológico se realizó mediante la observación directa por microscopia. Se analizaron 73.660 muestras, observándose huevos de $H$. nana en 158 pacientes ( 31 mujeres); media de 18,9 años de edad. La prevalencia fue de $0,21 \%$; $61 \%$ de los pacientes estaban poliparasitados. En conclusión, la prevalencia de parasitosis por $H$. nana en nuestra población fue mayor que la media nacional y mayor en adultos que en niños debido a las características de la población de nuestra área de influencia.

\section{Referencias bibliográficas}

1.- Crompton D W. How much human helminthiasis is there in the world. J Parasitol 1999; 85: 397-403.
2.- Schantz P M. Tapeworms (cestodiasis). Gastroenterol Clin North Am 1996; 25: 637-53.

3.- Macnish M. Characterisation of community-derived Hymenolepis infections in Australia. Murdoch University. Medical Science Thesis, 2001. Disponible: http://researchrepository.murdoch.edu.au/176/1/01Front.pdf

4.- Smythand J D, McManus D P. The Physiology and Biochemistry of Cestodes. Cambridge University Press. Cambridge, UK, 1989; p. 232.

5.- Hennessy T W, Marcus R, Deneen V, Reddy S, Vugia D, Townes J, et al. Survey of physician diagnostic practices for patients with acute diarrhoea: clinical and public health implications. Clin Infect Dis. 2004; 38 Suppl 3: S203-11.

6.- $\quad$ Liu L X, Weller P F. Antiparasitic Drug. N Engl J Med 1996; 334: 1178-84.

7.- $\quad$ Leder K, Weller P F. Intestinal tapeworm. Med Lett Drugs Ther 2010; 8: e20.

8.- Juan J O, López Chegne N, Gargala G, Favennec L. Comparative clinical studies of nitazoxanide, albendazole and praziquantel in the treatment of ascariasis, trichuriasis and hymenolepiasis in children from Peru. Trans R Soc Trop Med Hyg 2002; 96: 193-6.

9.- Chero J C, Saito M, Bustos J A, Blanco E M, Gonzalvez G, García H H. Hymenolepis nana infection: symptoms and response to nitazoxanide in field conditions. Trans R Soc Trop Med Hyg 2007; 101: 203-5.

10.- Orlandi P A, Chu D M T, Bier J W, Jackson G J. Parasites and the food supply. Food Technol 2002; 56: 72-81.

11.- D'Annibale M L, Bracciale S, Vitali M, Fonzo G, Verdini C, Papili R. Parasitic intestinal infections in human between 2006 and 2007. Microbiol Med 2009; 24: 19-24.

12.- Instituto Nacional de Estadística de España. Cifras de población y censos demográficos. Disponible: http://www.ine.es/inebmenu/mnu_cifraspob.htm (Fecha de acceso: 16 de enero de 2015).

13.- Federación Andaluza de Asociaciones Solidarias con el Sahara. http://www. saharandalucia.org/index.php/2013-05-30-13-07-43/vacaciones-en-paz (Fecha de acceso:16 de diciembre de 2014).

14.- Knott J. A method for making microfilarial surveys on day blood. Trans R Soc Trop Med Hyg 1939; 33:191-6.

15.- Mirdha B R, Samantray J C. Hymenolepis nana: a common cause of paediatric diarrhoea in urban slum dwellers in India. J Trop Pediatr 2002; 48: 331-4.

16.- Mason J B. Lessons on nutrition of displaced people. J Nutr 2002; 132 : 2096S-2103S

17.- Ahmed A M, Afifi A A, Malik E M, Adam I. Intestinal protozoa and intestinal helminthic infections among school children in Central Sudan. Asian Pac J Trop Med 2010; 3: 292-3.

18.- Borjas P, Arenas F, Angulo-Bazán Y. Enteroparasitismo en niños y su relación con la pobreza y estado nutricional. CIMEL 2009; 14 (1): 49-53.

19.- Crivello G, Fiddian E, Chatty D. Mobility and the care of Sahrawi refugee youth. Anthropology News 47 (5): 29-30.

20.- Soriano J M, Domènech G, Martínez M C, Mañes J, Soriano O F. Intestinal parasitic infections in hosted Saharawi children. Trop Biomed 2011; 28: 557-62. 